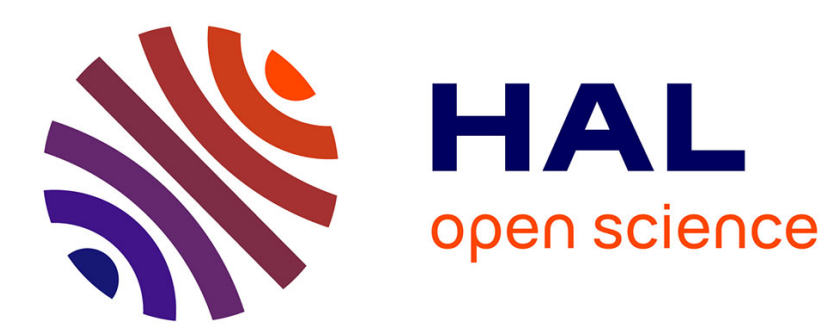

\title{
Generation of coherent pulses by the CDW motion. Interference effects
}

\author{
A. Bjelis, D. Jelčić
}

\section{To cite this version:}

A. Bjelis, D. Jelčić. Generation of coherent pulses by the CDW motion. Interference effects. Journal de Physique Lettres, 1985, 46 (6), pp.283-287. 10.1051/jphyslet:01985004606028300 . jpa-00232512

\section{HAL Id: jpa-00232512 https://hal.science/jpa-00232512}

Submitted on 1 Jan 1985

HAL is a multi-disciplinary open access archive for the deposit and dissemination of scientific research documents, whether they are published or not. The documents may come from teaching and research institutions in France or abroad, or from public or private research centers.
L'archive ouverte pluridisciplinaire HAL, est destinée au dépôt et à la diffusion de documents scientifiques de niveau recherche, publiés ou non, émanant des établissements d'enseignement et de recherche français ou étrangers, des laboratoires publics ou privés. 
Classification

Physics Abstracts

$72.15 \mathrm{~N}-72.20 \mathrm{H}$

\title{
Generation of coherent pulses by the CDW motion. Interference effects
}

\author{
A. Bjeliš (*) \\ Institute of Physics of the University, P.O.B. 304, 41001 Zagreb, Croatia, Yugoslavia
}

and D. Jelčić

Department of Physics, Faculty of Sciences, P.O.B. 162, 41001 Zagreb, Croatia, Yugoslavia

(Reçu le 22 octobre 1984, révisé le 17 janvier 1985, accepté le 28 janvier 1985)

\begin{abstract}
Résumé. - L'influence d'un champ électrique ac sur les phénomènes de transport collectif des ondes de densité de charge est analysée par l'intermédiaire du modèle de Gor'kov sur les oscillations de courant. Dans la limite asymptotique des champs forts $(E \gtrsim 1)$, les résonances sont trouvées aux valeurs $E / m$ i.e. pour les sous-harmoniques de la fréquence du fondamental. On affirme que dans le domaine expérimental des champs faibles $(E \ll 1)$, le modèle donne les résonances pour toutes les valeurs rationnelles $n E / m$ ainsi qu'elles ont été observées dans $\mathrm{NbSe}_{3}$.
\end{abstract}

\begin{abstract}
The influence of the external ac electric field on the collective charge density wave transport is considered within Gor'kov's model of current oscillations. In the asymptotic limit of large dc fields $(E \gtrsim 1)$ the resonances are found at $E / m$, i.e. at the subharmonics of the fundamental frequency. It is claimed that in the experimental range of fields $(E \ll 1)$ the model gives resonances at all rational values $n E / m$, as was observed recently in $\mathrm{NbSe}_{3}$.
\end{abstract}

The current oscillations induced by the collective charge density wave (CDW) transport is today the well-known phenomenon, observed in transition metal trichalcogenides like $\mathrm{NbSe}_{3}$ and $\mathrm{TaS}_{3}$ and in some other materials [1]. In last years a considerable amount of work was done aiming to determine the mechanism responsible for this " periodic noise ». Particularly important in this direction are experiments $[2,3]$ which indicate that the current oscillations are generated locally, at the contact of samples. In spite of actual controversies regarding some of these evidences [3-5], they strongly support the idea $[6,7]$ that the "periodic noise " originates from the conversion of the CDW transport to the ohmic one in the region of contacts.

This conversion is inevitable whenever a defect in the sample is so strong that the whole threedimensionally correlated CDW is pinned. Obviously, the contacts represent such an example. Then the motion of CDW may be stopped by both the mechanical strains and the local drop of the electrical field below the threshold field $E_{\mathrm{T}}$ due to shunting of the current across the probes.

(*) Temporary address : Laboratoire de Physique des Solides, Université Paris-Sud, 91405 Orsay Cedex, France. 
Gor'kov [6,7] envisaged such a situation [8] by defining the bordering surface $x=0$ with a fixed value of the order parameter,

$$
\Delta(x=0, t)=\Delta_{0}, \quad\left|\Delta_{0}\right| \geqslant 1,
$$

where the dimensionless complex CDW amplitude $\Delta(x, t)$ is measured in terms of $\left|\Delta_{\infty}\right|=$ $\left(\frac{6}{5} \pi^{2} T_{\mathrm{p}}^{2}\left(1-T^{2} / T_{\mathrm{p}}^{2}\right)\right)^{1 / 2}$, and $T_{\mathrm{p}}$ is the critical temperature. The CDW motion is allowed only on one side, say $x>0$, and is determined by the equation of motion

$$
\frac{\mathrm{d} \Delta}{\mathrm{d} t}-\frac{\mathrm{d}^{2} \Delta}{\mathrm{d} x^{2}}+i E \Delta-\Delta+|\Delta|^{2} \Delta=0
$$

derived on the microscopic grounds discussed in detail in reference [7]. All quantities in equation (2) are dimensionless, i.e. the electrical field $E$, position $x$ and time $t$ are measured in terms of $\pi^{2} T_{\mathrm{p}}^{2}\left(1-T^{2} / T_{\mathrm{p}}^{2}\right)^{1 / 2} / 6 \bar{v}_{x} e, \xi_{\|}$and $\omega_{0}^{-1}$ respectively. $\xi_{\|} \sim \frac{\hbar v_{\mathrm{F}}}{T_{\mathrm{p}}}$ is the longitudinal correlation length, and $\bar{v}_{x}$ is the mean longitudinal electron velocity. The frequency $\omega_{0}$ is given by

$$
\omega_{0}=\frac{16 \pi \gamma}{27} \cdot \frac{T_{\mathrm{p}}^{2}}{T_{\mathrm{p}}^{\mathrm{o}}}\left(1-T^{2} / T_{\mathrm{p}}^{2}\right)^{1 / 2},
$$

where $T_{\mathrm{p}}^{\mathrm{o}}$ is the Peierls transition temperature in absence of impurities, and $\gamma$ is the Euler constant. Gor'kov's model assumes an impurity concentration close to the critical one, so that $T_{\mathrm{p}}>T_{\mathrm{p}}^{\mathrm{o}}$.

Equation (2) was treated analytically in the limit $E \gtrsim 1[6,7]$, and numerically in the more interesting range $E \ll 1$ [9] which corresponds to the experimental electric fields (the field of $100 \mathrm{mV} / \mathrm{cm}$ equals to $E \simeq 10^{-4}$ ). The central result is that the condition (1) leads to the formation of the phase slippages (PS) in the plane $x_{\mathrm{PS}} \simeq E^{-0.284}$. The PSs repeat periodically in time, and last in time intervals much shorter than the period $2 \pi / E$. The distance of PS centres from the border $x=0$ is in the limit $E \ll 1$ much larger than the unit length (i.e. the correlation length $\xi_{\|} \simeq 10^{-6} \mathrm{~cm}$ ). Thus, the properties of PSs should not be too sensitive to the details of the contact, such as its geometry, roughness etc. This was indeed confirmed numerically [9].

The fast variation of $\Delta(x, t)$ at the PS centres leads to the finites periodic pulses in the CDW current density $[6,7]$

$$
j_{\mathrm{CDW}}=\frac{\sigma_{\mathrm{n}} \pi^{2} T_{\mathrm{p}}^{2}\left(1-T^{2} / T_{\mathrm{p}}^{2}\right)^{1 / 2}}{6 \bar{v}_{x} e}\left[i \frac{8}{9} \varepsilon \lambda\left(\dot{\Delta} \Delta^{*}-\Delta \dot{\Delta}^{*}\right)-\lambda E|\Delta|^{2}\right],
$$

and in the induced voltage proportional to the length integral of the bracket in equation (4). Here $\varepsilon=\bar{v}_{x}^{2} / \bar{v}_{x}^{2}$, and $\lambda \sim \frac{T_{\mathrm{p}}^{2}}{T_{\mathrm{p}}^{\mathrm{o}}}\left(1-T^{2} / T_{\mathrm{p}}^{2}\right)$.

Since $\lambda \ll 1, j_{\text {CDw }}$ and the corresponding fluctuations in the local electrical field are much weaker than the ohmic current and the external electric field $E$ respectively.

The Fourier transform of $j_{\mathrm{CDw}}$ contains harmonics at $\omega=n E$ which, for $E \lesssim 1$, diminish slowly with increasing $n$ [9]. As $E$ increases this decrease becomes faster, and finally in the limit $E \gtrsim 1$, equation (4) approaches sinusoidal behaviour, so that only the fundamental harmonic at $\omega=E$ remains [7]. These results are in agreement with the data obtained in the direct spectral analysis of the "periodic noise " in $\mathrm{NbSe}_{3}$ [10-12] and $\mathrm{TaS}_{3}$ [13].

Another method of analysing the periodic noise was initiated by Monceau et al. [11, 12], who applied the electric field with a finite ac component,

$$
E(t)=E+E_{1} \cos \left(\omega_{0} t+\phi_{0}\right)
$$


and measured the resulting differential resistance in $\mathrm{NbSe}_{3}$ as a function of $\omega_{0}$. In these, as well as in the subsequent measurements of the differential resistance versus dc current $[14,15]$ (i.e. dc field $E$ ), the resonant peaks were found at both, harmonics ( $\omega_{0}=n E$ in our notation) and subharmonics $\left(\omega_{0}=E / m\right)$ of the fundamental frequency $E$. These peaks correspond to the Shapiro steps observed in $I-V$ characteristics $[14,15]$ at the same values of $E$.

It has been reported recently [5] that the magnitudes of Shapiro steps are independent of the sample length, and that they also do not scale with the intensity of periodic noise which varies from sample to sample. This would indicate that the Shapiro steps are by themselves generated locally, but however contradicts the conclusion that two effects are of the same origin, which follows from e.g. the comparison of the resonance measurements [12] and the analysis of periodic noise [3]. In this Letter we assume that the data of reference [11-15] represent resonances between the external ac field and the periodic noise, and make a preliminary attempt to evaluate these resonances in the frame of Gor'kov's model [1-4]. This is to be contrasted to the proposal [16] that the Shapiro steps are produced by imposing the ac field on the deformable CDW in motion and are not directly connected with the generation of periodic noise.

We start by solving equation (2) with the field (5) in which $E \gtrsim 1$. Following the procedure of [7], the solution can be constructed by matching particular solutions in two ranges, $x \gtrsim 1$ and $x \ll 1, \Delta=\Delta_{\mathrm{R}}+\Delta_{\mathrm{L}} \cdot \Delta_{\mathrm{R}}(x, t)$ is the propagating CDW in the sample interior, which ends as a kink at $x=0$,

$$
\Delta_{\mathrm{R}}(x, t)=\exp \left[-i \int_{t} E\left(t^{\prime}\right) \mathrm{d} t^{\prime}\right] \tanh (x / \sqrt{2})
$$

$\Delta_{\mathrm{L}}(x, t)$ is expected to vary strongly on the $x$-scale, and to vanish on moving away from the fixed end. Then, the last two terms in equation (2) (and in particular the cubic one) are small. We therefore linearize (2), and get the solution (with the boundary condition (1) and $\Delta_{0}=1$ ).

$$
\Delta_{\mathrm{L}}(x, t)=\exp \left[-i \int_{t} E\left(t^{\prime}\right) \mathrm{d} t^{\prime}\right] \sum_{m} \exp \left[i\left(E-m \omega_{0}\right) t-i m \phi_{0}+i k_{m} x\right] J_{m}\left(-E_{1} / \omega_{0}\right),
$$

where the summation goes over all integers, and $k_{m}=\left[i\left(m \omega_{0}-E\right)+1\right]^{1 / 2}$ with $\operatorname{Im} k_{m}>0$.

The solution (7) clearly shows a resonance behaviour for $\omega_{0}=E / m$. Being the solution of the linear equation, $\Delta_{L}(x, t)$ then does not vanish even for $x \rightarrow \infty$. (The remaining unessential oscillating factor exp $(i x)$ comes from retaining of the term $\Delta(x, t)$ in Eq. (2)). The original non linearity of the problem would however modify this behaviour, so that close to resonances $\left(\left|m \omega_{0}-E\right| \lesssim \sqrt{2}\right)$ the factor $\exp \left(-\operatorname{Im} k_{m}\right)$ in $(7)$ is to be replaced by the faster one, $\exp (-\sqrt{2} x)$ modulated by some smooth function of $x$. Note also that equation (7) is valid for all interesting values of $E_{1}$, providing $E \gtrsim 1$. Namely, for $E_{1} \lesssim 1$ the total field (5) is always large, while for $E_{1} \gtrsim E$ and in the resonance range of frequencies $(\omega \lesssim E)$ the Bessel functions in (7) are much smaller than unity. This justifies the linearization of equation (2) for $x \lesssim 1$.

The time dependence of the complete solution $\Delta(x, t)$ defined by equations (6) and (7) is characterized by two frequencies, $E$ and $\omega_{0}$. The same dependence is present in its zeros, $\Delta\left(x_{\mathrm{PS}}, t_{\mathrm{PS}}\right)=0$, which determine the positions and moments of PSs. In other words, both, $x_{\mathrm{PS}}$ and the time interval between two successive PSs (which is equal to $2 \pi / E$ for $E_{1}=0$ ) are modulated periodically with the frequency $\omega_{0}$. Only for the ratio $\omega_{0} / E$ equal to an integer or a reciprocal integer, PSs attain the one periodic behaviour. This becomes evident after evaluating the induced local electrical field $E_{\mathrm{CDW}}(x, t)$ given by the expression inside the square brackets in equation (4). Here we keep only the most important contribution, i.e. that one in which $E$ and $\omega_{0}$ oscillations 
interfere in the PS range of $x$ :

$$
\begin{aligned}
E_{\mathrm{CDW}}(x, t) \simeq & \tanh \left(\frac{x}{\sqrt{2}}\right) \sum_{m}\left\{E J_{m}\left(-E_{1} / \omega_{0}\right) \cos \left[\operatorname{Re} k_{m} x-m \phi_{0}+\left(E-m \omega_{0}\right) t\right] \mathrm{e}^{-\operatorname{lm} k_{m} x}+\right. \\
& \left.+E_{1} \sum_{ \pm} J_{m \pm 1}\left(-E_{1} / \omega_{0}\right) \cos \left[\operatorname{Re} k_{m \pm 1} x-m \phi_{0}+\left(E-m \omega_{0}\right) t\right] \mathrm{e}^{-\operatorname{lm} k_{m \pm 1} x}\right\} .
\end{aligned}
$$

The most important property of the result (8) is the appearance of a finite, $\phi_{0}$-dependent dc contribution to $E_{\mathrm{CDW}}(x, t)$ whenever the ratio $E / \omega_{0}$ passes through an integer value. The expression (8) ressembles in this sense to those usually used in the description of Shapiro steps in Josephson junctions [17]. In order to find a corresponding step in the voltage, we integrate the dccomponent of $E_{\mathrm{CDW}}(x, t)$ along $x$, and chose a value of $\phi_{0}$ which gives the maximum value of the step. The resulting step heights at $E=m \omega_{0}$ are given by

$$
\delta V_{m} \simeq\left\{\left[2 \alpha E J_{m}\left(-E_{1} / \omega_{0}\right)\right]^{2}+\left(\frac{E_{1}}{\omega_{0}}\right)^{2}\left[J_{m+1}\left(-E_{1} / \omega_{0}\right)-J_{m-1}\left(-E_{1} / \omega_{0}\right)\right]^{2}\right\}^{1 / 2},
$$

where the factor $\alpha$ is of the order of unity and depends on the detailed form of $\Delta_{\mathrm{L}}(x, t)$ for $E=m \omega_{0}$.

The solutions (6) and (7) of the model (2) in the limit of extremely large dc fields $(E \gtrsim 1)$ gives rise to the Shapiro steps (and to the corresponding peaks in the differential resistance) at the subharmonics of the fundamental frequency $\left(\omega_{0}=E / m\right)$, while no steps are obtained at harmonic $\left(\omega_{0}=n E\right)$ and rational $\left(\omega_{0}=\frac{n}{m} E\right)$ values of external frequency. However, it is to be expected that in the physical range of fields $(E \ll 1)$ the latter steps would be present as well. It was already mentioned that the production of PSs at $E \ll 1$ has a rich multiharmonic content [9], in contrast to the limit $E \gtrsim 1$ analysed here, in which only a basic harmonic $(E)$ remains [7]. The straightforward extension of the problem defined by equations (1), (2) and (5) to the range of low dc fields $(E \ll 1)$ would therefore presumably lead to the appearance of additional terms with higher harmonics of $E$ in the expression (8). The presence of phase factors $\left(n E-m \omega_{0}\right) t$ in these terms would then give a complete set of Shapiro steps at $E=\frac{m}{n} \omega_{0}$, as was observed experimentally $[15,5]$.

The extrapolation to low dc fields will also very probably modify the $E, E_{1}$ and $\omega_{0}$ dependence of step heights given by equation (9) (modifications of that kind are present already for $E_{1}=0$; e.g. the amplitude of the first harmonic in the PS-voltage is $E$-independent for $E \gtrsim 1$ and behaves like $E^{\mathbf{0 . 5 6}}$ for $E \ll 1$ [9]). Nevertheless, we note that some correct trends, like the increase of $\delta V_{m}$ with increasing the frequency $\omega_{0}$ [14] and with decreasing the interference number $m$ [12], are present already in equation (9). Furthermore, the appearance of the term proportional to $J_{m+1}-J_{m-1}$ beside the usual one, $J_{m}$, makes the expression (9) different from that obtained within the model of rigid CDW [14]. This expression is expected to have the same structure for $E \ll 1$. The additional term in equation (9) becomes more and more important as $E_{1}$ increases, so that it may improve the fit of $V v s . E_{1}$ and $V v s . \omega_{0}$ dependences for $E_{1} \gtrsim E$ [14], and explain the apparent random variation of peak amplitudes in the differential resistance at high ac fields [5]. For the quantitative analysis in this direction, it is however necessary to extrapolate the expression $(9)$ to the physical range of field $(E \ll 1)$, as was already mentioned.

In conclusion, we analysed the interference effects in the asymptotic $E \gtrsim 1$ limit of the Gor'kov's model $[6,7]$, and found finite Shapiro steps at the subharmonic values of the fundamental frequency of periodic noise, $E / \omega_{0}=m$. Taking into account the previous numerical results for the PS voltage in the absence of ac field [9], we expect that the extrapolation of the present results 
to experimental values of dc field $(E \ll 1)$ would give also the steps at $E / \omega_{0}=m / n$. These latter steps were interpreted [18] within the rigid CDW model as the consequence of the finite inertial term $\mathrm{d}^{2} \Delta / \mathrm{d} t^{2}$, which is however expected to be extremely small in the real systems $[1,12]$. We note that there is no need for such term in equation (2) which is substantially more complex than the differentional equation for the rigid CDW dynamics $[14,18]$, and describes the process of non linear diffusion of PSs in the vicinity of contacts. As was shown in the numerical analysis [9], this process becomes more and more complex as the dc field $E$ decreases [6, 7]. It is not clear at present whether the finite external ac field in equation (5) would lead to additional effects in this regime, like e.g. the period doubling route to chaos in the vicinity of interferences, observed recently in $\mathrm{NbSe}_{3}$ [19]. The further investigations in this direction are in progress.

\section{Acknowledgments.}

We are grateful to I. Batistić for helpful suggestions and to S. Barišić, S. Coppersmith and J. Friedel for useful discussions. The work is partially supported by the Yu-US collaboration contract DOE 438.

\section{References}

[1] For references see :

(a) Review article of GrüNER, G. and ZETTL, A., to be published ;

(b) Proc. of the Int. Symp. on Nonlinear Transport and Related Phenomena in Organic Quasi One Dimensional Conductors, October 1983 (Hokkaido University, Sapporo, Japan) 1983.

[2] Gill, J. C. and HigGS, A. W., Solid State Commun. 48 (1983) 709.

[3] Ong, N. P. and Verma, G., Phys. Rev. B 27 (1983) 4495 :

Ong, N. P., Verma, G. and Maki, K., Phys. Rev. Lett. 52 (1984) 663.

[4] Mozurkewich, G. and Grüner, G., Phys. Rev. Lett. 51 (1983) 2206.

[5] Hall, R. P. and Zettl, A., Phys. Rev. B 30 (1984) 2279.

[6] GoR'kov, L. P., Pis'ma Zh. Eksp. Teor. Fiz. 38 (1983) 76.

[7] GoR'kov, L. P., Zh. Eksp. Teor. Fiz. 86 (1984) 1818.

[8] The role of contacts was invoked independently by Ong et al. (Ref. [3] ; see also K. Maki, Ref. [1b], p. 17), who proposed that the periodic noise originates from the non uniform motion of phase vortices towards contacts.

[9] Batistić, I., Bueliš, A. and Gor'kov, L. P., J. Physique 45 (1984) 1049.

[10] Fleming, R. M. and Grimes, G. G., Phys. Rev. Lett. 42 (1979) 1423.

[11] Monceau, P., Richard, J. and Renard, M., Phys. Rev. Lett. 45 (1980) 43.

[12] Richard, J., Monceau, P. and Renard, M., Phys. Rev. B 25 (1982) 948.

[13] Grüner, G., Zettl, A., Clark, W. G. and Thompson, A. H., Phys. Rev. B 23 (1981) 6813.

[14] Zettl, A. and Grüner, G., Solid State Commun. 46 (1983) 501 ; Phys. Rev. B 29 (1984) 755.

[15] Brown, S. E., Mozurkewich, G. and Grüner, G., Phys. Rev. Lett. 52 (1984) 2277.

[16] Sneddon, L., Cross, M. C. and Fisher, D. S., Phys. Rev. Lett. 49 (1982) 292.

[17] See Shapiro, E. G. S., Janus, A. R. and Holly, S., Rev. Mod. Phys. 36 (1964) 223 ; and KaUtz, R. L., J. Appl. Phys. 52 (1981) 3528.

[18] BaK, P., Ref. [1b], p. 13.

[19] Hall, R. P., Sherwin, M. and Zettl, A., Phys. Rev. B 29 (1984) 7076. 